\title{
Cross-reactivity of anti-human cytokine monoclonal antibodies used as a tool to identify novel immunological biomarkers in domestic ruminants
}

\author{
E.M.S. Dorneles ${ }^{1}$, M.S.S. Araújo ${ }^{2}$, A. Teixeira-Carvalho², \\ O.A. Martins-Filho ${ }^{2}$ and A.P. Lage ${ }^{1}$ \\ ${ }^{1}$ Laboratório de Bacteriologia Aplicada, \\ Departamento de Medicina Veterinária Preventiva, Escola de Veterinária, \\ Universidade Federal de Minas Gerais, Belo Horizonte, MG, Brasil \\ ${ }^{2}$ Laboratório de Biomarcadores de Diagnóstico e Monitoração, \\ Centro de Pesquisas René Rachou, Fundação Oswaldo Cruz, \\ Belo Horizonte, MG, Brasil \\ Corresponding author: A.P. Lage \\ E-mail: alage@vet.ufmg.br
}

Genet. Mol. Res. 14 (1): 940-951 (2015)

Received August 1, 2013

Accepted June 26, 2014

Published February 3, 2015

DOI http://dx.doi.org/10.4238/2015.February.3.1

\begin{abstract}
Eleven commercially available PE-labeled anti-human (IL-1- $\alpha$, IL-6, IL-8, TNF- $\alpha$, IL-17A, IL-5, IL-10, IL-12 and IL-13) and anti-mouse (IL-10, TNF- $\alpha$ ) cytokine monoclonal antibodies (mAbs) were tested for cross-reactivity with cattle, goat, and sheep cytokines. Cross-reactivity was assessed by comparative analysis with the standard reactivity of the target species. Our data demonstrated that anti-human IL-1- $\alpha$, IL-6, IL-8, IL-17A and IL-10 mAbs cross-react with all ruminant species tested. Anti-human IL-5 mAb showed a strong cross-reactivity with cattle and goat IL-5, while anti-human TNF- $\alpha$ mAb showed a selective cross-reactivity with goat TNF- $\alpha$. No cross-reactivity with the ruminant cytokines was observed for anti-human IL-12 and IL-13
\end{abstract}


mAbs or for the two anti-mouse cytokine mAbs tested. The present study demonstrated the cross-reactivity of various anti-human cytokine mAbs with cattle, sheep, and goat cytokines, increasing the range of immunological biomarkers for studies in veterinary medicine.

Key words: Cytokines; Cross-reactivity; Human; Cattle; Goat; Sheep

\section{INTRODUCTION}

Cytokines released from different cells activate an entire network of interactions between cells and occupy a strategic position in the development of immune responses. Among the numerous physiologic roles played by cytokines are the development of cellular and humoral immune responses, induction of inflammatory responses, regulation of hematopoiesis, control of cell proliferation and differentiation, and wound healing (Kindt et al., 2007).

Analysis of cytokines in biological fluids could be a useful tool in the diagnosis and in the understanding of pathologic conditions in domestic animals (Dernfalk et al., 2004). Specific monoclonal antibodies (mAbs) are essential for the assessment of intracellular cytokines by flow cytometry. However, studies on the role of cytokines and immune mechanisms involved in domestic ruminant diseases are severely hampered by the low availability or lack of species-specific reagents.

The search for cross-reactivity of commercially available monoclonal antibodies (mAbs) between different species has been suggested as a putative strategy to obtain valuable reagents for immunological studies in veterinary medicine. Numerous studies have been done to identify the existence of cross-reactivity among mAbs of different species by flow cytometry, especially the ability of antibodies directed to cytokines or surface markers of animal cells (Davis et al., 1987; Naessens et al., 1993; Brodersen et al., 1998; Griebel et al., 2007). Scheerlinck (1999) suggests a higher probability of occurrence of cross-reactivity when amino acid sequence homology between cytokines from different species is at least $60 \%$. The comparison of cytokine amino acid sequence of human/mouse and ruminant species have demonstrated up to $84 \%$ homology for a selected set of pro-inflammatory and regulatory cytokines, suggesting high probability of cross-reactivity among several anti-cytokine mAbs (Table 1).

Pedersen et al. (2002), investigating the existence of cross-reactivity of anti-ovine, -bovine and -human IL-2, IL-4, IL-6, IL-8, IL-12, TNF- $\alpha$, IFN- $\gamma$ and GM-CSF mAbs with sheep, cow, goat, pig, horse, dog, mink, and human antigens found biological cross-reactivity for the majority of the species investigated with four mAbs specific for IL-4, IL-8, IFN- $\gamma$, and TNF- $\alpha$.

Considering that a better understanding of the immune response in diseases can lead to the development of new diagnostic methods and vaccines and that the development and production of new monoclonal antibodies are expensive and slow, the aim of this study was to evaluate, by flow cytometry, the cross-reactivity of commercial anti-human/mouse cytokine mAbs with cytokines from cattle, goat, and sheep.

\section{MATERIAL AND METHODS}

\section{Monoclonal antibodies (mAbs)}

A total of eleven commercially available anti-cytokine mAbs conjugated with phyco- 
erythrin (PE) were used in this study. mAbs clone specifications, host/target species, immunoglobulin isotype and manufacturer are provided in Table 2.

\section{Animals and controls}

A total of fifteen healthy domestic ruminants were included in this investigation, including five cattle (Bos taurus), five goats (Capra hircus), and five sheep (Ovis aries). All animals were maintained at Fazenda Modelo (Escola de Veterinária, Universidade Federal de Minas Gerais (UFMG), located in Pedro Leopoldo, Minas Gerais State, Brazil. Ten milliliters of heparinized peripheral whole blood were collected from each domestic ruminant and maintained at room temperature up to $24 \mathrm{~h}$ prior to processing.

Four healthy human subjects (Homo sapiens) living in Belo Horizonte, Minas Gerais State, Brazil and three healthy Swiss mice (Mus musculus), maintained in the animal facility at Centro de Pesquisas René Rachou, FIOCRUZ-Minas were included as control groups to evaluate the standard reactivity of anti-human and anti-mouse cytokine mAbs with the target species, respectively. Ten milliliters of human heparinized peripheral whole blood were collected by venipuncture, whereas mouse blood was collected from the orbital sinus with a glass Pasteur pipette, immediately transferred to a polypropylene conical vial containing sodium heparin as anticoagulant and pooled. Human and mouse blood were also maintained at room temperature up to $24 \mathrm{~h}$ prior to processing.

This study was approved by the Ethical Committee for the Use of Experimental Animals (CEUA) of the Fundação Oswaldo Cruz, Brazil, and by the Ethical Committee for the Use of Experimental Animals of the Universidade Federal de Minas Gerais, Brazil (CETEA). All animal procedures were in accordance with the guidelines of the Brazilian Association for Laboratory Animal Science (COBEA). The inclusion of human subjects in this study complied with the regulations 196/1996 (Brasil, 1996) of the Brazilian National Council on Research in Humans and was approved by the Ethical Committee of the Fundação Oswaldo Cruz, Brazil.

\section{Short-term whole blood in vitro culture and immunostaining for intracellular cytokine analysis by flow cytometry}

Short-term whole blood in vitro culture and immunostaining for intracellular cytokine was adapted for ruminants, according to the protocol described by Teixeira-Carvalho et al. (2008) (Figure 1). Briefly, 1-mL aliquots of whole blood samples were cultured $\left(4 \mathrm{~h}\right.$ at $37^{\circ} \mathrm{C}$, $5 \% \mathrm{CO}_{2}$ ) in $1 \mathrm{~mL}$ RPMI 1640 (Invitrogen, USA), referred to as Control cultures. Other 1-mL aliquots were incubated under the same conditions, but cultured in $1 \mathrm{~mL}$ RPMI 1640 supplemented with $50 \mu \mathrm{L} 1 \mu \mathrm{g} / \mathrm{mL}$ phorbol 12-myristate 13-acetate (Sigma Aldrich, USA) plus $2 \mu \mathrm{L}$ $1 \mathrm{mg} / \mathrm{mL}$ ionomycin (Sigma Aldrich), referred to as PMA+IONO-stimulated cultures. Twenty microliters of $1 \mathrm{mg} / \mathrm{mL}$ brefeldin A (BFA) (Sigma Aldrich) were added to all whole blood cultures. Following short-term in vitro culture, $220 \mu \mathrm{L} 20 \mathrm{mM}$ EDTA were added to each tube before incubation for $10 \mathrm{~min}$ at room temperature. Erythrocytes were then lysed and leukocytes fixed with $3 \mathrm{~mL}$ FACS Lysing Solution (Becton Dickinson, USA). After centrifugation at 400 $g$ for $10 \mathrm{~min}$, the fixed leukocytes were incubated with $3 \mathrm{~mL}$ FACS permeabilizing buffer (permBuffer - $0.01 \mathrm{M}$ PBS, $\mathrm{pH} 7.2$, supplemented with $0.5 \%$ bovine serum albumin plus $0.5 \%$ 
saponin and $0.1 \%$ sodium azide, all from Sigma Aldrich) for $10 \mathrm{~min}$ at room temperature. Following this step, samples were incubated with $15 \mu \mathrm{L}$ permBuffer (Tube 1), $15 \mu \mathrm{L}$ PE-labeled anti-cytokine mAbs (Table 1) diluted 1:50 (dilution determined by previous experiments) in sterile permBuffer (Tube 2) or $15 \mu \mathrm{L}$ whole serum blocking buffer (permBuffer supplemented with 5\% mouse/rat serum, both from the animal facility at the Centro de Pesquisas René Rachou, FIOCRUZ-Minas) to monitor nonspecific binding (Tube 3). The cells were incubated in the dark for $30 \mathrm{~min}$ at room temperature and then washed once with $2 \mathrm{~mL}$ permBuffer followed by a washing with washBuffer (0.01 M PBS, $\mathrm{pH} 7.2$, supplemented with $0.5 \%$ bovine serum albumin and $0.1 \%$ sodium azide, all from Sigma Aldrich) by centrifugation at $400 \mathrm{~g}$ for $10 \mathrm{~min}$. After washing, the cells stained were fixed in $200 \mu \mathrm{L}$ FACS fix solution $(10 \mathrm{~g} / \mathrm{L}$ paraformaldehyde, $10.2 \mathrm{~g} / \mathrm{L}$ sodium cacodylate, $6.63 \mathrm{~g} / \mathrm{L}$ sodium chloride, $\mathrm{pH} 7.2$, all reagents from Sigma Aldrich) and the samples immediately used for flow cytometry acquisition or stored at $4^{\circ} \mathrm{C}$ up to $24 \mathrm{~h}$ prior acquisition.

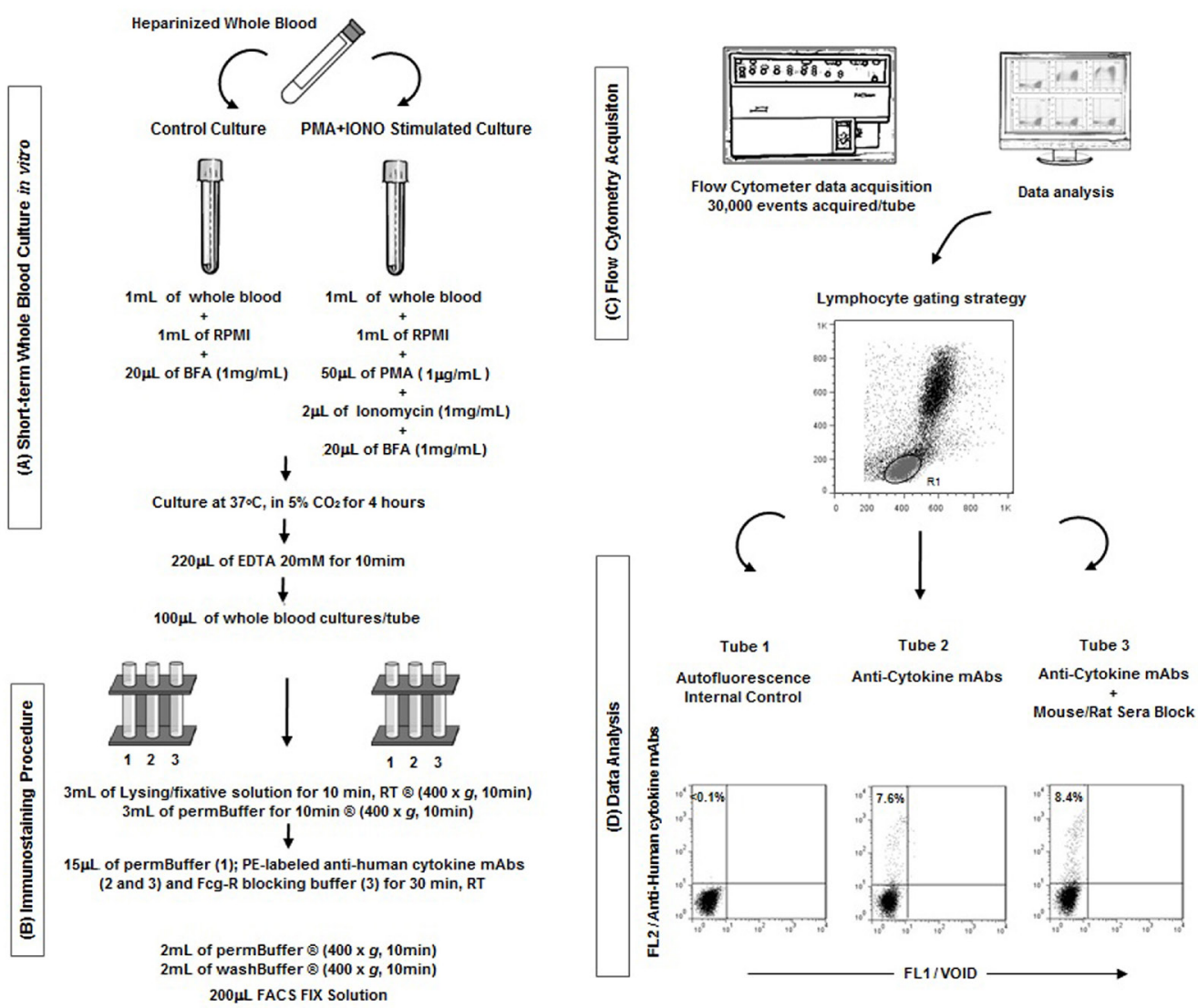

Figure 1. Short-term whole blood culture in vitro. Flowchart summarizing the major steps of (A) whole blood short-term culture in vitro, (B) the immunophenotyping procedures, (C) flow cytometry acquisition and (D) data analysis used to quantify the frequency of lymphocytes expressing intracytoplasmic cytokines. 


\begin{tabular}{|c|c|c|c|}
\hline Cytokine & Cattle (\%) & Sheep (\%) & Goat (\%) \\
\hline IL-1- $\alpha^{\mathrm{H}}$ & 71 & 70 & 72 \\
\hline IL-6 ${ }^{\mathrm{H}}$ & 53 & 52 & 53 \\
\hline IL-8 $8^{\mathrm{H}}$ & 79 & 81 & - \\
\hline IL-12 ${ }^{\mathrm{H}}$ & 82 & 80 & 80 \\
\hline TNF- $\alpha^{\mathrm{H}}$ & 80 & 79 & 80 \\
\hline IL-17A & 84 & 84 & 76 \\
\hline IL-5 ${ }^{\mathrm{H}}$ & 66 & 65 & 66 \\
\hline IL-10 $0^{\mathrm{H}}$ & 76 & 76 & 76 \\
\hline IL-13 ${ }^{\mathrm{H}}$ & 66 & 65 & - \\
\hline TNF- $\alpha^{\mathrm{M}}$ & 73 & 72 & 73 \\
\hline $\mathrm{IL}-10^{\mathrm{M}}$ & 70 & 70 & 71 \\
\hline
\end{tabular}

Source: <http://www.ncbi.nlm.nih.gov/BLAST/>. ${ }^{\mathrm{H} H u m a n}$ amino acid sequence; ${ }^{\mathrm{M}}$ Mouse amino acid sequence.

The evaluation of cross-reactivity with ruminant cytokines was conducted in three rounds. First, the pattern of reactivity of mAbs with target species (human and mouse) was assessed. In a second step, the cross-reactivity of these mAbs with ruminant cytokines was evaluated. Finally, we evaluated the effect of whole serum blocking on the cross-reactivity observed in the previous step.

\section{Flow cytometry data storage and analysis}

Flow cytometric measurements were performed on a FACScan instrument (Becton Dickinson) interfaced to an Apple G3 FACStation. The FlowJo 7.6.1 (Tree Star, USA) software was used in data analysis. A total of 30,000 events were acquired for each sample.

Figure 1 summarizes the major steps of whole blood short-term in vitro culture, the immunophenotyping procedures, flow cytometry acquisition, and data analysis. Lymphocyte gating was based on cell selection by forward scatter (FSC) versus side scatter (SSC) properties on dot plot distributions, where they were confined to a region of low size and complexity (R1) (Figure 2). Cytokine-expressing lymphocyte cell subpopulations were quantified using Fluorescence 1 (FL1)/VOID versus Fluorescence 2 (FL2)/anti-cytokine-PE dot plots, by setting quadrants to segregate FL2 positive and negative cells on the basis of negative control immunostaining. The results are given as percentage of lymphocytes that express the cytokine of interest. Cross-reactivity for mAbs was evaluated on the basis of the reactivity observed with the target species (human and mouse). Cytokines whose main sources are not lymphocytes were also investigated in other populations of leukocytes (data not shown).

Figure 2 displays the gating strategy used to identify the lymphocyte population on the basis of size and granularity morphometric features. After appropriate instrument settings in the flow cytometer (FSC - gain 1.5 in linear scale and SSC - 359 in linear scale and threshold around 200 in FSC parameter) the lymphocytes displayed a homogeneous distribution in both Control and PMA+IONO-stimulated cultures. PMA+IONO-induced neutrophil death leading to a typical change in the FSC versus SSC profile as compared to the control cultures. Neutrophils, representing a large cell cluster with SSC $^{\text {high }}$ profile $(>400)$ were clearly observed following short-term whole blood in vitro culture maintained under control conditions but were almost absent in the cattle and goat PMA+IONO-stimulated cultures (Figure 2). 


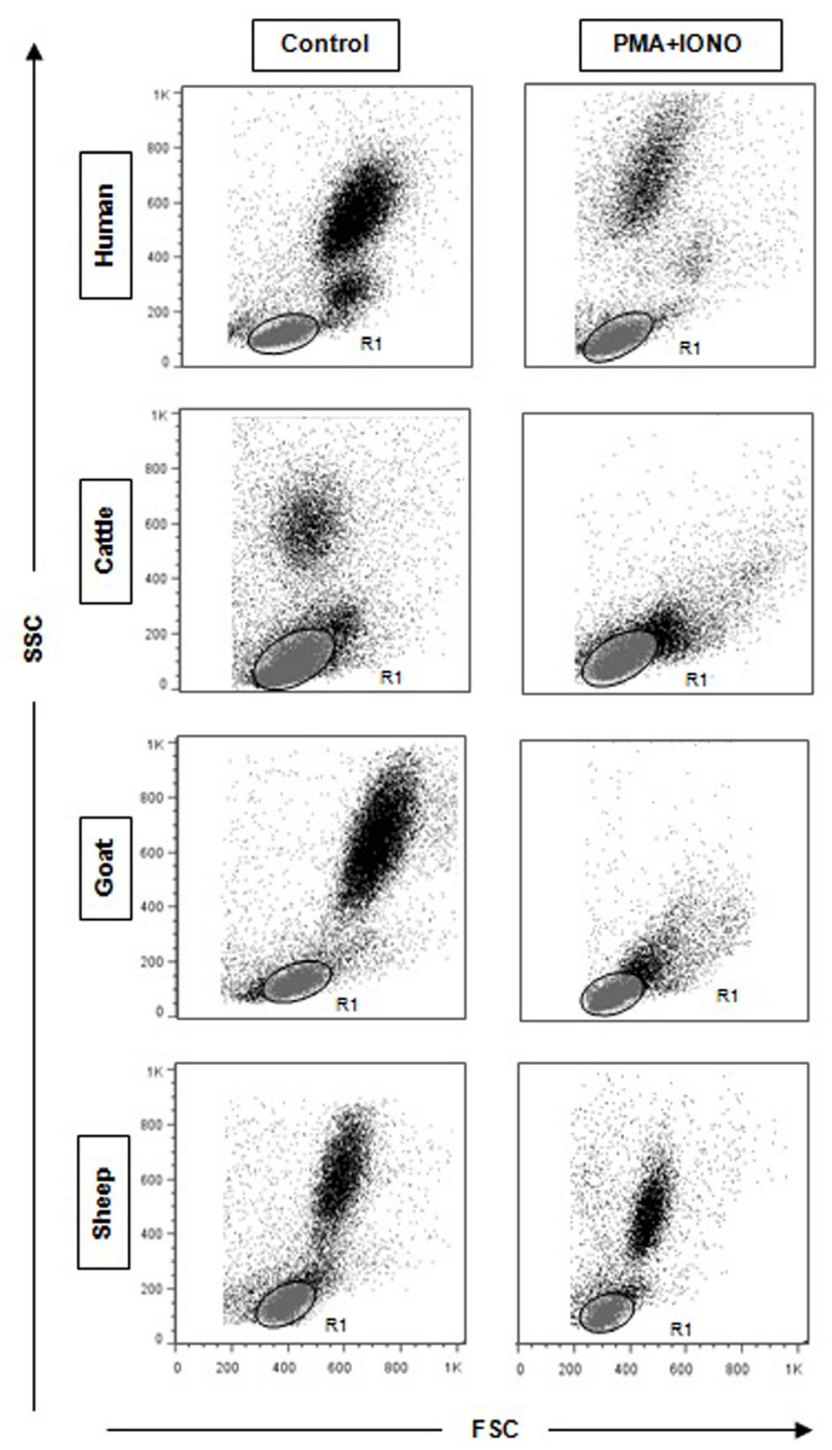

Figure 2. Gating strategy. Details of gating strategy used to select the lymphocyte population (R1) based on their size (Forward scatter - FSC) and granularity (Side scatter - SSC) flow cytometric features prior the analysis of cytokine + events. The lymphocytes assume a homogeneous distribution (gray ellipse) in Control cultures and PMA + IONO stimulated cultures. PMA + IONO stimuli (right panels) induced neutrophils death leading to a typical change in the FSC vs SSC profile as compared to the Control cultures (left panels).

\section{RESULTS}

Aiming to provide a range of valuable reagents for immunological studies in veterinary medicine, the present study accessed the patterns of reactivity of commercially available anticytokine mAbs with cytokines from domestic ruminants. For this purpose, a standard protocol 
for short-term whole blood in vitro culture was established and a flow cytometry-based immunofluorescence assay used to test the cross-reactivity pattern of eleven commercially available PElabeled anti-human cytokine (IL-1- $\alpha$, IL-6, IL-8, TNF- $\alpha$, IL-17A, IL-5, IL-10, IL-12, and IL-13), and anti-mouse cytokine (IL-10, TNF- $\alpha$ ) mAbs with cattle, goat, and sheep cytokines. Basal cytokine and PMA+IONO-induced cytokine profiles were evaluated and the cross-reactivity with ruminant cytokines assessed by comparative analysis with the standard reactivity of the target species (human and mouse). Data analysis demonstrated that five of eleven anti-human cytokine mAbs (IL-1- $\alpha$, IL-6, IL-8, IL-17A, and IL-10) cross-react with all ruminant species tested. The anti-human IL-5 mAbs showed cross-reactivity with cattle and goat IL-5. On the other hand, the anti-human TNF- $\alpha$ mAb showed a selective cross-reactivity with goat TNF- $\alpha$ (Figure 3 ).

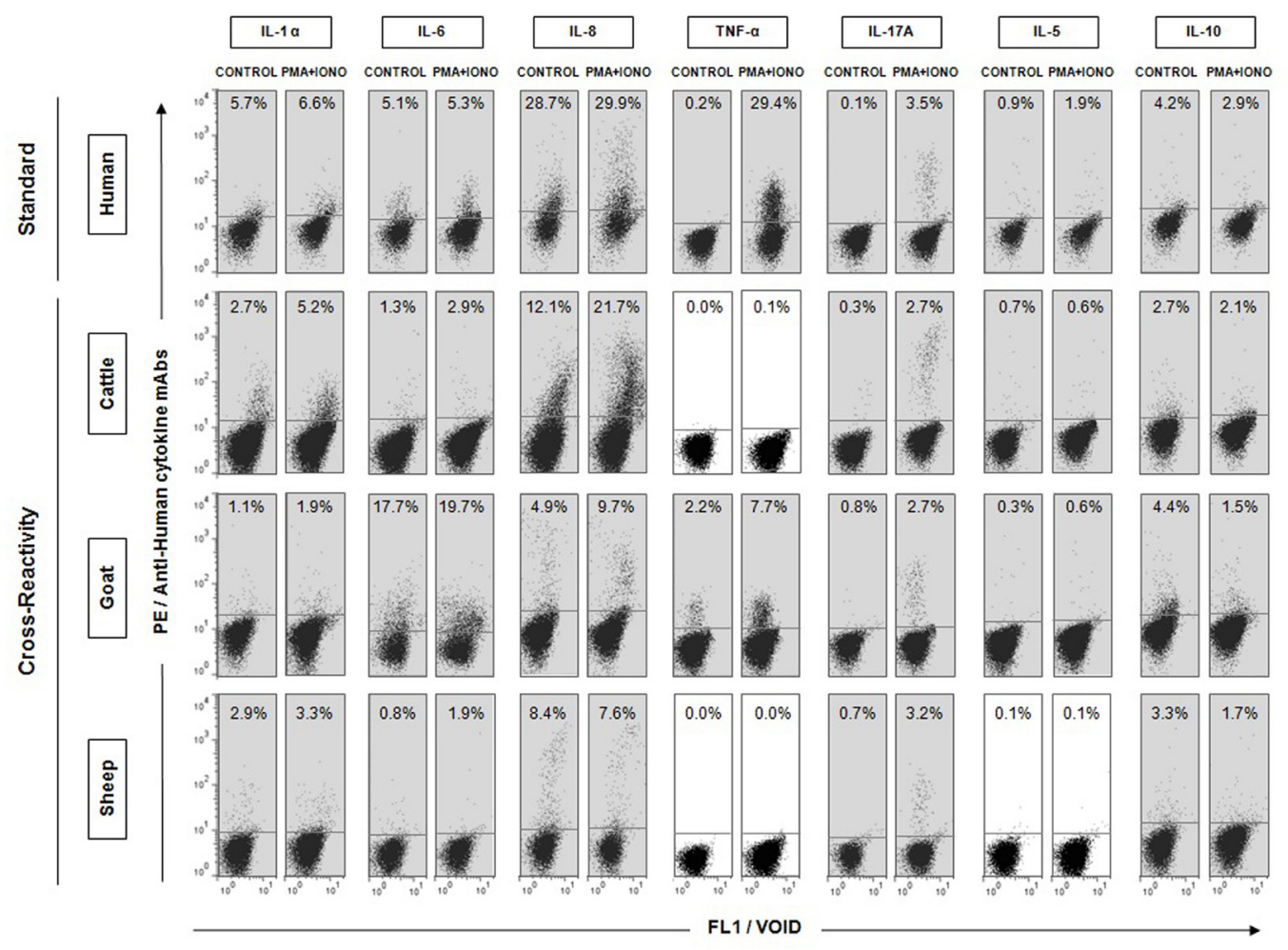

Figure 3. Cross-reactivity of anti-human cytokine mAbs with cattle, goat, and sheep lymphocytes. Flow cytometric charts illustrating the standard reactivity of anti-human cytokine mAbs (IL-1- $\alpha$, IL-6, IL-8, IL-17A, TNF- $\alpha$, IL-5, IL10) with human lymphocytes and the cross-reactivity with cattle, goat and sheep lymphocytes. Short-term whole blood culture in vitro was performed in the absence (Control) and in the presence of PMA plus Ionomycin (PMA+IONO) as described in Material and Methods. After the immunostaining procedures and flow cytometric acquisition, lymphocytes were first selected based on their homogeneous morphometric profile on size versus granularity dot plot distribution. Following gating strategies, dual-quadrant statistics were used to quantify the frequency of cytokineexpressing lymphocytes on FL1 versus FL2 dot-plot distribution, using the autofluorescent internal control as the baseline for the cutoff definition (gray line). The results are expressed as percentage of cytokine+ events within gated lymphocytes. The standard anti-human cytokine mAbs profile and the cross-reactivity patterns are highlighted by gray rectangles. The dot plot distributions are representative of five independent experimental batches. Standard reactivity with human samples was monitored by four independent experimental batches. 
The overall pattern of cross-reactivity observed for the anti-human and anti-mouse $\mathrm{mAbs}$, besides the clone specifications for all mAbs, and the manufacturer of the commercially available reagents as well are summarized in Table 2 . The selected set of mAbs with outstanding cross-reactivity with ruminant cytokines is highlighted by gray shading.

\begin{tabular}{|c|c|c|c|c|c|c|c|}
\hline \multirow[t]{2}{*}{$\mathrm{mAb^{ \textrm {a } }}$} & \multirow[t]{2}{*}{ Target species } & \multirow[t]{2}{*}{ Clone } & \multirow[t]{2}{*}{ Host } & \multirow[t]{2}{*}{ Isotype } & \multicolumn{3}{|c|}{ Cross-reactivity pattern } \\
\hline & & & & & Cattle & Goat & Sheep \\
\hline Anti-IL-1 $\alpha$ & Human & $3643 \mathrm{~B} 314^{\mathrm{b}}$ & Mouse & $\operatorname{IgG1}$ & + & + & + \\
\hline Anti-IL-6 & Human & MQ2-13A5 & Rat & IgG1 & + & + & + \\
\hline Anti-IL-8 & Human & $\mathrm{G} 265-8^{\mathrm{b}}$ & Mouse & $\mathrm{IgG} 2 \mathrm{~b}$ & + & + & + \\
\hline Anti-IL-12 & Human & $\mathrm{C} 11.5 .14^{\mathrm{b}}$ & Mouse & IgG1 & - & - & - \\
\hline Anti-TNF- $\alpha$ & Human & Mab11 & Mouse & IgG1 & - & + & - \\
\hline Anti-IL-17A & Human & eBio64DEC17 ${ }^{c}$ & Mouse & IgG1 & + & + & + \\
\hline Anti-IL-5 & Human & 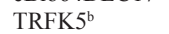 & Rat & IgG1 & + & + & - \\
\hline Anti-IL-10 & Human & JES3-19F $1^{\mathrm{b}}$ & Rat & $\mathrm{IgG} 2 \mathrm{a}$ & + & + & + \\
\hline Anti-IL-13 & Human & JES10-5A2 $2^{b}$ & Rat & IgG1 & - & - & - \\
\hline Anti-TNF- $\alpha$ & Mouse & MP6-XT22 & Rat & IgG1 & - & - & - \\
\hline Anti-IL-10 & Mouse & JES5-16E3 $3^{b}$ & Rat & $\operatorname{lgG} 2 b$ & - & - & - \\
\hline
\end{tabular}

${ }^{a} \mathrm{mAb}$ - monoclonal antibody; ${ }^{\mathrm{b}} \mathrm{mAb}$ purchased from BD Pharmingen (USA); ${ }^{\mathrm{c}} \mathrm{mAb}$ purchased from eBiosciense (USA). The set selected of mAbs with outstanding cross-reactivity with ruminant cytokines is marked by shaded areas.

To further address whether the cross-reactivity of the selected set of anti-human cytokine mAbs with ruminant cytokines would represent nonspecific binding via Fcy-R, we performed parallel immunophenotyping staining in the "absence" or in the "presence" of mouse/ rat serum reagent. This strategy was used to provide a major source of unlabeled IgG for species-specific whole serum blocking by competitive binding. Due to the lack of universal cross-reactivity, the anti-human TNF- $\alpha$ and the anti-human IL-5 mAbs were selectively used to monitor the nonspecific binding with goat and cattle/goat lymphocytes, respectively. Our data demonstrated that there were no differences in the "presence" of "whole serum blocking" as compared with the reference immunophenotyping procedure performed in the "absence" of "whole serum blocking" (Figure 4).

\section{DISCUSSION}

Cross-reactivity studies using well-defined mAbs are an important and quick way to obtain reagents that will be valuable for studies on domestic animal immunology, since the development and production of new mAbs is time-consuming and expensive (Dernfalk et al., 2004). The present study identified seven mAbs against anti-human cytokines (Table 2) that cross-reacted with cattle, sheep, or goat cytokines, which can improve the information on immunological status and host-parasite relationship in these species.

A general problem in cytokine flow cytometry concerns the establishment of cut-off levels between positive and negative cross-reactivity (Pedersen et al., 2002). To avoid this problem, cross-reactivity for each cytokine mAb was assessed by comparison with the staining profile in the target species, used as standard reactivity. Five healthy individuals of each domestic ruminant species were used in assays to add more reliability to the findings. Moreover, the lack of cells producing a particular cytokine was minimized through the use of short-term whole blood culture that results in smaller loss of cells due to cell adherence or during cell isolation, 
which occurs in assays with peripheral blood mononuclear cells (PBMC). Furthermore, the observed cross-reactivity patterns were very consistent for all animals from the same species studied. Additionally, the time of culture in this study $(4 \mathrm{~h})$ was chosen on the basis of previously reported kinetic studies that found a decrease in cytokine production after long incubation with brefeldin A (Sewell et al., 1997; Mascher et al., 1999). PMA, a known activator of protein kinase $\mathrm{C}$, and ionomycin were chosen as stimulants because the stimulus was deliberately supraphysiological in an attempt to activate as many cells as possible, mainly primed cells, capable of producing a given cytokine. However, the extent that PMA induces cytokine generation does vary between cytokines and depends on the cell source (Schauer et al., 1996).

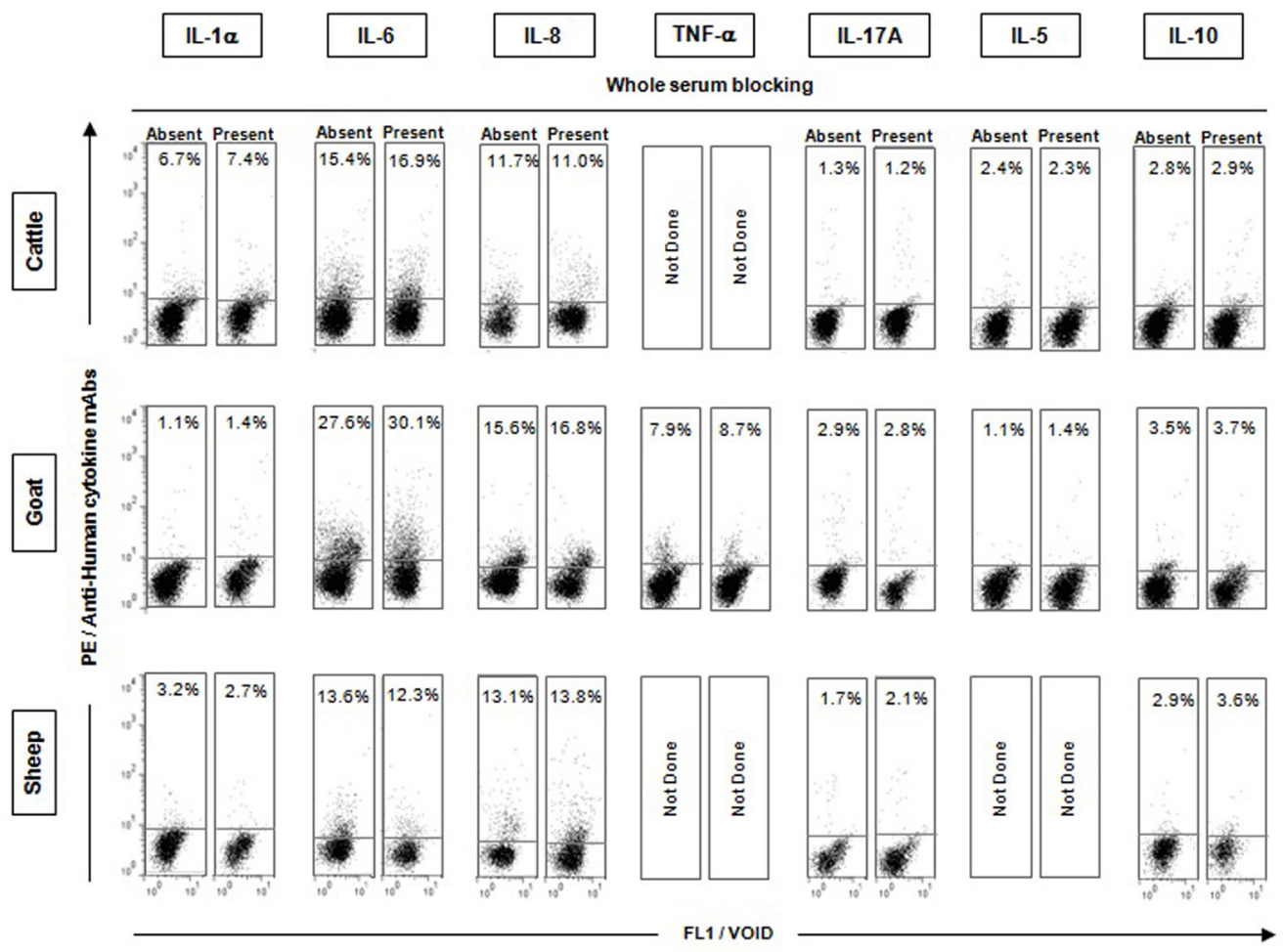

Figure 4. Whole serum blocking. Flow cytometric charts illustrating no impact of "Whole serum blocking" with mouse/rat sera reagent on the binding profile of anti-human cytokines mAbs (IL-1- $\alpha$, IL-6, IL-8, TNF- $\alpha$, IL-17A, IL10 , and IL-5) with cattle, sheep, and goat lymphocytes. Comparative analysis of FL1 versus FL2 dot plot distribution showed that the blocking procedure led to no changes in the binding profile of anti-human cytokines mAbs observed after short-term whole blood culture in vitro. The results illustrated the cytokine profile of gated cattle, goat, and sheep lymphocytes, following in vitro stimulation with PMA+IONO. The results are expressed as percentage of cytokine + events within gated lymphocytes. Due to the lack of universal cross-reactivity, the anti-human TNF- $\alpha$ and the anti-human IL-5 mAbs were selectively used to monitor the unspecific binding with goat and cattle/goat lymphocytes, respectively. No significant differences were observed in the "presence" of "whole serum blocking" (right panels) as compared with the reference immunophenotyping procedure performed in the "absence" of "whole serum blocking" (left panels). The dot plot distributions are representative of two independent experimental batches.

Our results showed that anti-human IL-1 $\alpha$, IL-8, IL-17A, and IL-10 mAbs cross-reacted with the three ruminant species studied. On the other hand, anti-IL-5 mAb exhibited 
cross-reactivity with bovine and goat cytokines, and TNF- $\alpha$ mAb showed cross-reactivity only with goat cytokines. These results are supported by a high degree of amino acid sequence homology found between human, cattle, sheep, and goat IL-1 $\alpha$, IL-5, IL-8, IL-17A, IL-10, and TNF- $\alpha$ (Table 1), which showed greater than $65 \%$ amino acid sequence similarity. Similarly, anti-human IL-6 mAb also displayed cross-reactivity with all domestic ruminants studied; however, the degree of similarity for IL-6 amino acid sequence between human, cattle, sheep, and goat was smaller. Despite the low similarity (around 50\%), it is probable that the epitope recognized by this $\mathrm{mAb}$ remained evolutionarily conserved among these species, explaining the cross-reactivity observed. The findings with "whole serum blocking", confirmed the potential of this cross-reactivity study to enhance the tools available for immunological assays in veterinary medicine.

These cross-reactivity results are broadly supported by the findings in "whole serum blocking" testing, which confirmed the link of the mAbs tested as the Fab portion. Moreover, the cross-reactivity found in the present study is an important tool in determining the contribution of different cells to cytokine production in heterogeneous cell populations, since surface markers are more widely available than anti-cytokines for domestic ruminant species. Additionally, IL-1 $\alpha$, IL-6, IL-8, and TNF- $\alpha$ are important pro-inflammatory cytokines, and the clear cross-reactivity found in this study provides new tools to evaluate the production of these cytokines in domestic ruminants. Also, the cross-reactivity of anti-human IL-17A mAb opens a new horizon to investigate the $\mathrm{T}_{\mathrm{H}} 17$ cells, which require a unique combination of cytokines and depend on distinct intracellular events (Hirota et al., 2010), in the immune response of domestic ruminants. Additionally, IL-5 and IL-10, a $\mathrm{T}_{\mathrm{H}} 2$ and regulatory cytokine, respectively, are also very important for understanding the immunological status in domestic ruminants, especially under pathologic conditions. These cytokines showed the lowest frequency of positive cells, and for IL-10 this percentage is even lower under the stimulus of PMA+IONO. Both results can be easily understood, not because of difficulty finding a suitable mAb, but because these cytokines need restimulation in vitro to increase their frequency of response (Sander et al., 1993; Schauer et al., 1996; Caraher et al., 2000). This was especially evident for IL-10-secreting cells, which increased 30-80 times in secondary responses (Sander et al., 1993; Caraher et al., 2000), in which secretion may have been inhibited in the presence of PMA (Boehringer et al., 1999).

Corroborating the present findings, Pedersen et al. (2002) found cross-reactivity between anti-ovine IL-8 and cattle leukocytes. Furthermore, the identification of cross-reactivity for mAbs against TNF- $\alpha$ between evolutionarily related species has been difficult (Dernfalk et al., 2004; Kwong et al., 2010). The findings of cattle and sheep cytokines against antihuman TNF- $\alpha$ were similar to those reported by Dernfalk et al. (2004). Using the same clone for TNF- $\alpha$ (Mab 11), they were also unable to detect ovine or bovine TNF- $\alpha$, although $80 \%$ homology is found between human and bovine TNF- $\alpha$, and ovine and bovine TNF- $\alpha$ show more than 90\% homology of nucleotide sequences (Dernfalk et al., 2004). Kwong et al. (2010) produced the mAbs CC327 and CC328 by immunizing mice, which were used to develop a sandwich ELISA capable of detecting both native and recombinant bovine TNF- $\alpha$; however, only CC328 detected intracellular ovine TNF- $\alpha$. Thus, high homology of nucleotide or amino acid sequence alone does not define the occurrence of cross-reactivity, which requires the recognition epitope of the mAb tested to be conserved in the different species in question.

Therefore, the absence of cross-reactivity between anti-human IL-12 and IL-13 mAbs 
with the respective cytokines produced by cells of the domestic ruminants tested is easily understood. The deficient evolutionary conservation of epitopes among species probably also caused the absence of cross-reactivity observed for anti-mouse IL-10 and TNF- $\alpha$ in all ruminant species tested. Since the amino acid identity between mouse IL-10 and TNF- $\alpha$ and the ruminant species is greater than $70 \%$ (Table 1) and since both anti-mouse and anti-human IL10 were produced in the same host (rat), the cross-reactivity differences can be attributed only to differences in the specificities of each $\mathrm{mAb}$.

Maintenance of epitopes on molecules may reflect the functional importance of the region of the molecule recognized and an evolutionary pressure to conserve those regions (Sopp and Howard, 2001). Comparison of the cytokine amino acid sequences (http://www. ncbi.nlm.nih.gov/BLAST/) between the control (human/mouse) and ruminant species studied (Table 1) showed that they can share up to $84 \%$ homology, which suggests a higher probability of occurrence of cross-reactivity (Scheerlinck, 1999). However, some results found in this study and in others (Pedersen et al., 2002; Dernfalk et al., 2004) do not completely support this theory, probably because the epitopes recognized by $\mathrm{mAbs}$ are not necessarily in regions that have been evolutionarily conserved. Comparative studies of the reactivity with cells from several species have shown that each antibody recognizes a different epitope (Davis et al., 1987). Weynants et al. (1998) tested various monoclonal antibodies against ovine IL-4, but found only one antibody with satisfactory reactivity. Thus, it is often necessary that several antibodies against the same antigen should be tested, which could suggest that a cross-reacting $\mathrm{mAb}$ could be found if other different clones were tested.

The present study demonstrated a range of anti-human cytokine mAbs that crossreacted with cattle, sheep and goat cytokines, expanding the scope of immunological biomarkers for studies on pathologic conditions in veterinary medicine. The mAbs that cross-reacted with cattle, sheep and goat cytokines in flow cytometry provide very useful tools that could be used to understand the regulation of immune response in healthy and unhealthy animals and that also allow not only the identification and quantification of the cytokine produced but the characterization of the cell population that produced it.

\section{ACKNOWLEDGMENTS}

Research supported by Conselho Nacional de Desenvolvimento Científico e Tecnológico, Fundação de Amparo à Pesquisa do Estado de Minas Gerais - Fapemig and FEP - MVZ Coordenação Preventiva. The authors thank the Program for Technological Development in Tools for Health - PDTIS - FIOCRUZ for use of its facilities. The authors are extremely grateful to Dr. A.S. Guimarães, Prof. E.J. Facury Filho, Prof. A.U. Carvalho and residents of the Clinic for Ruminants of Escola de Veterinária-UFMG for their essential technical assistance in this study. EMSD, ATC, APL and OAMF thank the Conselho Nacional de Desenvolvimento Científico e Tecnológico - CNPq for the fellowships.

\section{REFERENCES}

Boehringer N, Hagens G, Songeon F, Isler P, et al. (1999). Differential regulation of tumor necrosing factor-alpha (TNF-alpha) and interleukin-10 (IL-10) secretion by protein kinase and phosphatase inhibitors in human alveolar macrophages. Eur. Cytokine Netw. 10: 211-218.

Brasil (1996). Ministério da Saúde, Conselho Nacional de Saúde, Resolução nº 196 de 10 de Outubro de 1996. Aprova as 
Seguintes Diretrizes e Normas Regulamentadoras de Pesquisas Envolvendo Seres Humanos.

Brodersen R, Bijlsma F, Gori K, Jensen KT, et al. (1998). Analysis of the immunological cross reactivities of 213 well characterized monoclonal antibodies with specificities against various leucocyte surface antigens of human and 11 animal species. Vet. Immunol. Immunopathol. 64: 1-13.

Caraher EM, Parenteau M, Gruber H and Scott FW (2000). Flow cytometric analysis of intracellular IFN-gamma, IL-4 and IL-10 in CD3(+)4(+) T-cells from rat spleen. J. Immunol. Methods 244: 29-40.

Davis WC, Marusic S, Lewin HA, Splitter GA, et al. (1987). The development and analysis of species specific and cross reactive monoclonal antibodies to leukocyte differentiation antigens and antigens of the major histocompatibility complex for use in the study of the immune system in cattle and other species. Vet. Immunol. Immunopathol. 15: 337-376.

Dernfalk J, Waller KP and Johannisson A (2004). Commercially available antibodies to human tumour necrosis factor- $\alpha$ tested for cross-reactivity with ovine and bovine tumour necrosis factor-alpha using flow cytometric assays. Acta Vet. Scand. 45: 99-107.

Griebel PJ, Entrican G, Rocchi M, Beskorwayne T, et al. (2007). Cross-reactivity of mAbs to human CD antigens with sheep leukocytes. Vet. Immunol. Immunopathol. 119: 115-122.

Hirota K, Martin B and Veldhoen M (2010). Development, regulation and functional capacities of Th17 cells. Semin. Immunopathol. 32: 3-16.

Kindt TJ, Goldsby RA and Osborne BA (2007). Kuby Immunology. 6th ed. W.H. Freeman and Company, New York.

Kwong LS, Thom M, Sopp P, Rocchi M, et al. (2010). Production and characterization of two monoclonal antibodies to bovine tumour necrosis factor alpha $(\mathrm{TNF}-\alpha)$ and their cross-reactivity with ovine TNF- $\alpha$. Vet. Immunol. Immunopathol. 135: 320-324.

Mascher B, Schlenke P and Seyfarth M (1999). Expression and kinetics of cytokines determined by intracellular staining using flow cytometry. J. Immunol. Methods 223: 115-121.

Naessens J, Olubayo RO, Davis WC and Hopkins J (1993). Cross-reactivity of workshop antibodies with cells from domestic and wild ruminants. Vet. Immunol. Immunopathol. 39: 283-290.

Pedersen LG, Castelruiz Y, Jacobsen S and Aasted B (2002). Identification of monoclonal antibodies that cross-react with cytokines from different animal species. Vet. Immunol. Immunopathol. 88: 111-122.

Sander B, Höidén I, Andersson U, Möller E, et al. (1993). Similar frequencies and kinetics of cytokine producing cells in murine peripheral blood and spleen. Cytokine detection by immunoassay and intracellular immunostaining. $J$. Immunol. Methods 166: 201-214.

Schauer U, Jung T, Krug N and Frew A (1996). Measurement of intracellular cytokines. Immunol. Today 17: 305-306.

Scheerlinck JP (1999). Functional and structural comparison of cytokines in different species. Vet. Immunol. Immunopathol. 72: 39-44.

Sewell WA, North ME, Webster AD and Farrant J (1997). Determination of intracellular cytokines by flow-cytometry following whole-blood culture. J. Immunol. Methods 209: 67-74.

Sopp P and Howard CJ (2001). IFN- $\gamma$ and IL-4 production by CD4, CD8 and WC1 $\gamma \delta \mathrm{TCR}^{+} \mathrm{T}$ cells from cattle lymph nodes and blood. Vet. Immunol. Immunopathol. 81: 85-96.

Teixeira-Carvalho A, Martins-Filho OA, Peruhype-Magalhaes V, Silveira-Lemos D, et al. (2008). Cytokines, chemokine receptors, $\mathrm{CD}^{+} \mathrm{CD} 25^{\mathrm{HIGH}}$ T-cells and clinical forms of human schistosomiasis. Acta Trop. 108: 139-149.

Weynants V, Gilson D, Furger A, Collins RA, et al. (1998). Production and characterisation of monoclonal antibodies specific for bovine interleukin-4. Vet. Immunol. Immunopathol. 66: 99-112. 